\title{
Real-Time Optical Monitoring of Etching Reaction of Microfluidic Channel Fabricated by Femtosecond Laser Direct Writing
}

\author{
João M. Maia, Vítor A. Amorim, Daniel Alexandre, and Paulo V. S. Marques
}

\begin{abstract}
Femtosecond laser direct writing is a threedimensional fabrication technique that can be applied to produce integrated optical components with high spatial resolution or microfluidic channels when combined with HF etching. The same fabrication technique can thus be employed to produce monolithic optofluidic devices for sensing applications. One of the most common sensing schemes involves evanescent optical interaction; therefore, the channel must meet some requirements regarding surface roughness, which will depend on the laser writing conditions, as described in this paper. However, of more significance is the distance between waveguiding medium and microfluidic channel that must be accurately defined. This control can be achieved by monitoring the etching reaction of a waveguide grating written a few microns from the channel, as introduced in this paper. In addition to its function as an etching monitor, the grating can also be used as a coarse refractive index sensor device.
\end{abstract}

Index Terms-Etching monitorisation, femtosecond laser direct writing, optofluidics, refractive index sensor.

\section{INTRODUCTION}

$\mathbf{L}$ AB-ON-A-CHIP (LOC) devices allow the realization of many functions, ranging from preparation and transport of reagents to their analysis, in a hand-sized chip [1]. These systems comprise many components such as microfluidic channels (for fluid movement), mechanical elements (valves and mixers for control of reagent flow and reaction [2]) and optical elements (lenses and waveguides for reactant analysis [3]). These systems have become of high demand in basic research and medical

Manuscript received November 24, 2016; revised January 23, 2017; accepted February 27, 2017. Date of publication March 5, 2017; date of current version April 20, 2017. This work was supported under Project "NanoSTIMA: Macroto-Nano Human Sensing Towards Integrated Multimodal Health Monitoring and Analytics/NORTE-01-0145-FEDER-000016" financed by the North Portugal Regional Operational Programme (NORTE 2020), under the PORTUGAL 2020 Partnership Agreement, and through the European Regional Development Fund (ERDF).

J. M. Maia, V. A. Amorim, and P. V. S. Marques are with the Department of Physics and Astronomy, Faculty of Sciences, University of Porto, Porto 4169-007, Portugal, and also with the CAP - Centre for Applied Photonics, INESC TEC, Porto 4150-179, Portugal (e-mail: joao.m.maia@inesctec.pt; vitor.a.amorim@inesctec.pt; psmarque@fc.up.pt).

D. Alexandre is with the Department of Physics, University of Trás-os-Montes e Alto Douro, Vila Real 5001-801, Portugal, and also with the CAP - Centre for Applied Photonics, INESC TEC, Porto 4150-179, Portugal (e-mail: daniel@utad.pt).

Color versions of one or more of the figures in this paper are available online at http://ieeexplore.ieee.org.

Digital Object Identifier 10.1109/JLT.2017.2678604 applications, due to the possibility of replacing massive benchtop equipment by portable devices [4]. One of the key points in the development of such systems is thus, having a technique that enables integration of both optical layers with microfluidic channels for on-chip optical sensing. Typically, these devices are produced by lithography-based techniques [5], but can also be fabricated by femtosecond laser micromachining [6].

The latter technology has the advantage of allowing threedimensional (3D) fabrication and monolithical integration of different components, features that are not usually associated with conventional techniques [7]. This technique relies on a non-linear absorption process induced by pulsed laser irradiation, which modifies the material properties around the focal volume by: (i) increasing the refractive index to form waveguides and optical components [8]; or (ii) forming birefringent sub-wavelength nanogratings. These structures turn the initially isotropic etching reaction of fused silica into an anisotropic one, which allows formation of the microfluidic channels [9].

Usually, optofluidic systems analyse sample volumes of the order of $10^{-9}-10^{-12}$ litres, where surface forces (surface tension and roughness) become dominant and may impact the channel shape and function [10]. Smooth sidewall channels are important to decrease coupling losses upon integration with optical systems, while rougher channels may be of use for mixing different solutions [11]. Circular channels are preferable for mimicking the environment in blood vessels, while rectangular cross-sections are better for optical waveguide integration for cell trapping and stretching applications [12]. Therefore, the fabrication process of microfluidic channels must be accurately controlled.

Moreover, to detect small refractive index fluid changes efficiently it is necessary that the optical mode interacts with the solution in the channel. In the case of evanescent probing devices (either by surface plasmon resonance or by interferometry [13]) both components must be typically distanced by less than a couple of microns. In this paper, a technique for careful positioning of the optical waveguides relative to the microchannel is introduced, which involves analysis of the transmission/reflection spectrum of a waveguide containing a Bragg grating during the channel etching reaction. It should be noticed that with the aforementioned scheme, the optical layer besides serving as an etching sensor, it also functions as a coarse fluid refractometer sensor after etching. 


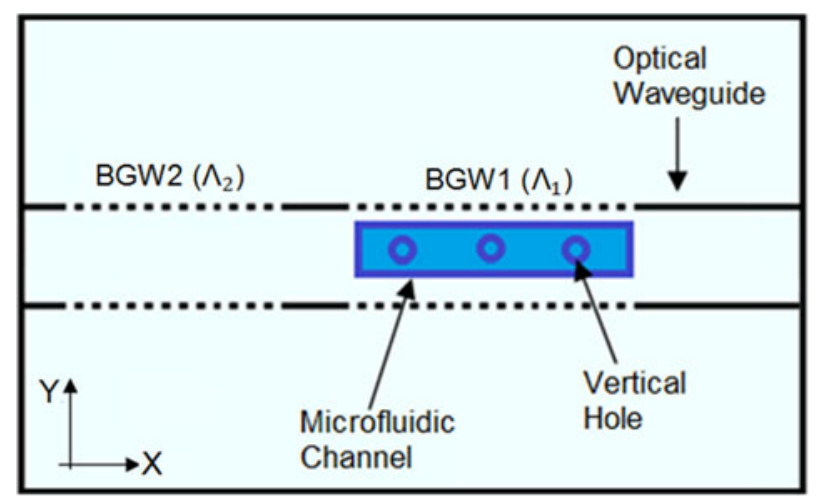

Fig. 1. Optofluidic device layout.

\section{DEVICE FABRICATION}

\section{A. Device Layout and Operation}

The fabricated and tested optofluidic device, shown in Fig. 1, is composed by a microfluidic channel and a waveguiding structure formed parallel to the channel.

The waveguiding medium is formed by two straight first order Bragg grating waveguides (BGWs) with different modulation periods $\left(\Lambda_{1}\right.$ and $\left.\Lambda_{2}\right)$.

Broadband light propagating in the waveguide is reflected, in a narrow range of wavelengths, due to the presence of the BGWs. According to the Bragg condition (1), the reflected wavelength $\left(\lambda_{B}\right)$ depends on the effective refractive index $\left(n_{\text {eff }}\right)$.

$$
\lambda_{B}=2 n_{\mathrm{eff}} \Lambda .
$$

Although the propagating light is confined to the waveguiding structure, there is an evanescent tail that enables light coupling. If the BGW1 is placed closely to the microfluidic channel, its effective index varies, which leads to a change in the Bragg wavelength, meaning that light can sense the properties of the fluid that is circulating inside the channel.

Since the effective index also depends on external parameters, such as room temperature, a second grating (BGW2) is fabricated outside the channel region to work as reference.

\section{B. Experimental Setup}

The device was fabricated in a pure fused silica substrate by femtosecond laser direct writing followed by chemical etching [14]. In the first step (femtosecond laser direct writing), the laser beam is focused inside the sample, changing its properties, either by increasing refractive index or by generating etching selectivity due to the formation of birefringent sub-wavelength nanogratings [15]. In the second step (chemical etching) the sample is immersed in a hydrofluoric acid (HF) solution that removes the laser-affected volumes at a higher rate than that of the pristine material, thus forming the microfluidic channels.

The laser direct writing unit is depicted in Fig. 2. A fiberamplified laser, from Amplitude Systèmes, generates laser pulses at $1030 \mathrm{~nm}$ (and also harmonics at $515 \mathrm{~nm}$ and 343 $\mathrm{nm})$ with $\sim 250$ fs duration and with repetition rates up to $2 \mathrm{MHz}$. In all experiments radiation at $515 \mathrm{~nm}$ with a $500 \mathrm{kHz}$

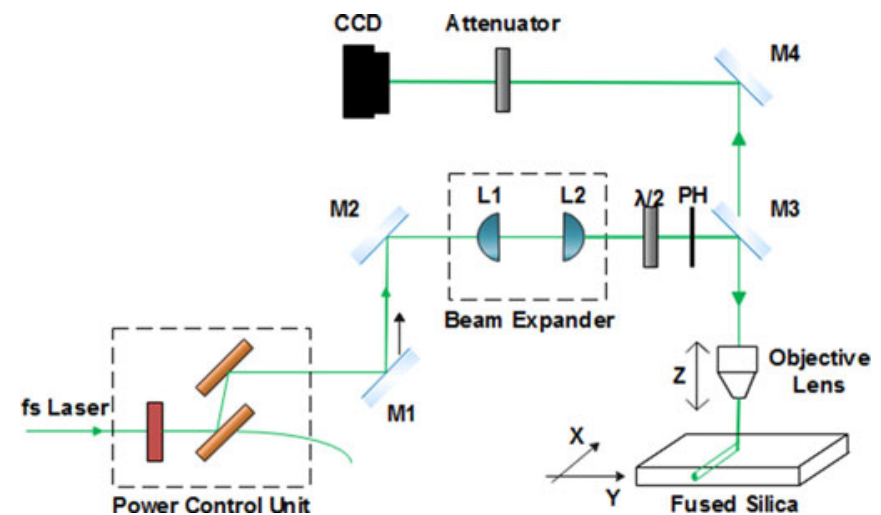

Fig. 2. Laser direct writing unit: $\lambda / 2=$ Half-wave plate, $A P=$ Aperture, $C C D$ $=$ Charge-coupled device, $\mathrm{L}=$ Lens, $\mathrm{M}=$ Mirror.

pulse repetition rate was used. The beam power is controlled by an external modulator (Watt Pilot unit, Altechna). In addition, a laser internal acousto-optic cell is driven by a function generator (Stanford Research Systems DS345) to act as a laser beam switch, which enabled the fabrication of BGWs. After crossing a beam expander and a half-wave plate (used to rotate the beam linear polarization), the laser pulse is focused inside the silica substrate by a 40x objective lens (numerical aperture $\mathrm{NA}=0.55$ ). The lens is mounted in a piezoelectric stage (PI $\mathrm{P}-725)$ that moves along $\mathrm{Z}$ while the sample is mounted in two air-bearing stages (Aerotech ABL 10100-LN) that move along the plane XY.

Both BGW and microfluidic channel are written in the same writing step. Although the BGW can be fabricated after etching, this approach would require highly accurate repositioning of the laser beam and the presence of the channel would cause significant border effects that could lead to channel damaging. The structures are written by translating the sample orthogonally to the laser beam. In this approach, the lens working distance does not impose any limitation on the device length, but its cross-section is asymmetric (usually elliptic) due to the different lateral and axial resolutions. The laser tracks are written always in the same direction (positive $\mathrm{X}$ axis from Fig. 2) to avoid quill effect [16].

To form microfluidic channels a multi-scanning technique is used, where the laser-affected zones are stacked side-by-side with a separation of few microns. With this method it is possible to obtain microfluidic channels with different cross-sections, depending on its application; in this paper only channels with rectangular cross-section were fabricated. The laser tracks are stacked from bottom-to-top to avoid propagation of the laser beam through already modified volumes.

After direct writing, the sample edges are polished to improve light coupling and fused silica V-groove blocks (Precision Micro-Optics) with two parallel-fibers with a $250 \mu \mathrm{m}$ pitch are glued to the sample with Norland Optical Adhesive 61. The glue is cured with an ultraviolet light source (Hamamatsu LC8 L9588-02). The etching reaction is then performed by immersing the sample in a diluted HF solution. In choosing the HF concentration, there is a trade-off between channel aspect ratio (length over cross-section dimension) and etch rate: although 


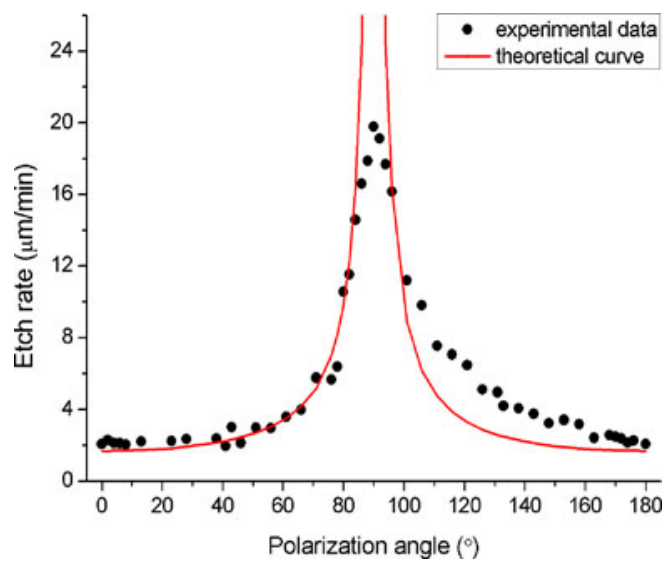

Fig. 3. Etch rate vs polarization angle of single-scan channels. The channels were written $150 \mu \mathrm{m}$ below the silica substrate surface with a beam with polarization perpendicular to the scanning direction, at $500 \mu \mathrm{m} / \mathrm{s}$ and $60 \mathrm{~nJ}$.The theoretical curve was obtained by assuming that the etch rate $(\mathrm{R})$ should change with the nanogratings orientation, according to the equation $\mathbf{R}=\mathbf{R}_{\|} \sec (\theta)$, where $\mathbf{R}_{\|}$is the etch rate for parallel polarisation and $\boldsymbol{\theta}$ is the angle between scanning direction and beam polarisation [19].

higher concentrations favour a faster etching reaction, they are usually accompanied by a saturation effect that significantly damages the channel aspect ratio; thus a $10 \% \mathrm{HF}$ concentration is used. The reaction is also performed in an ultrasonic bath (Branson 2510 Ultrasonic Cleaner) to facilitate debris removal and to avoid formation of bubbles within the channel.

\section{Fabrication and Calibration of Microfluidic Channels}

For the proposed device, it is necessary that the channel aspect ratio remains constant, as any variation in this parameter will lead to a variable distance between the BGW and the channel, which results in the broadening of the reflected peak. In addition, the channel sidewalls must be optically smooth to enable light coupling and to decrease optical losses due to scattering. Therefore, a detailed study on the effect of the irradiation conditions in the formation of microfluidic channels was performed in order to obtain smooth-walled channels with constant aspect ratio.

The channel etch rate (which affects the aspect ratio) and surface roughness were analysed for multiple channels fabricated under different irradiation conditions: beam polarization (parallel or perpendicular to the laser beam direction), pulse energy ( $60 \mathrm{~nJ}$ to $300 \mathrm{~nJ}$ ), scanning speed (100 $\mu \mathrm{m} / \mathrm{s}$ to $500 \mu \mathrm{m} / \mathrm{s}$ ), scanning depth $(50 \mu \mathrm{m}$ to $150 \mu \mathrm{m})$ and scan separation $(1 \mu \mathrm{m}$ to $15 \mu \mathrm{m})$.

As seen in Fig. 3, when the beam polarization is orthogonal to the scanning direction $\left(90^{\circ}\right)$, the etch rate is maximum because the nanogratings align with the channel axis, enabling fast HF diffusion [17]. For parallel polarization $\left(0^{\circ}\right.$ and $\left.180^{\circ}\right)$, the nanograting planes are perpendicular to the channel axis, hence the HF acid encounters alternate layers of nanogratings and pristine material, which reduce the etch rate. There is a 5 -fold decrease in the etch rate when varying the polarization angle from $90^{\circ}$ to $70^{\circ}$. Therefore, to take advantage of a maximum

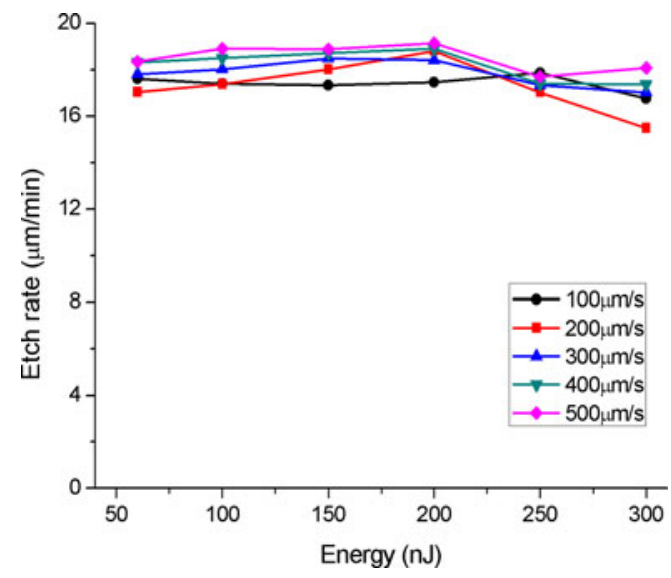

Fig. 4. Etch rate of single-scan channels as a function of scanning speed and pulse energy. The channels were written $150 \mu \mathrm{m}$ below silica surface with beam polarization perpendicular to the scanning direction.

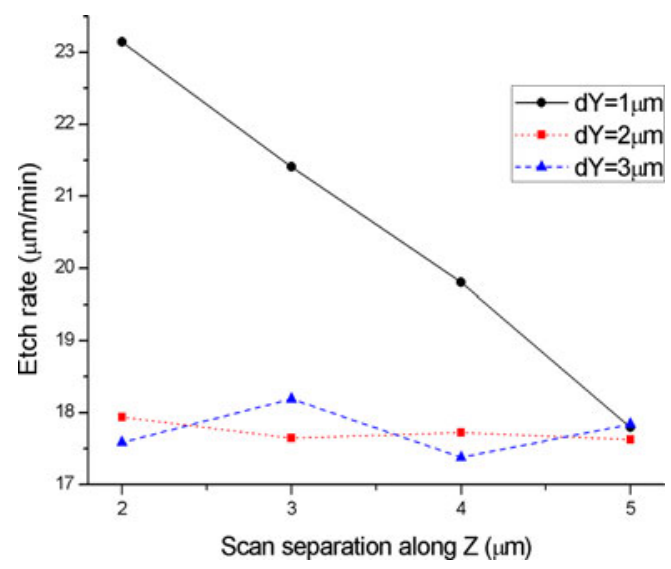

Fig. 5. Etch rate as a function of the separation between scans along Y (dY) and $\mathrm{Z}$ (dZ) respectively (according to the axis of Fig. 2).

etch rate, the polarization must be accurately oriented normal to the scanning direction.

In some applications, it might be advantageous to use circular polarization as it results in an angle-invariant etch rate due to the disordered orientation of the nanogratings [18].

It was also observed that the etch rate remained uniform within the tested range of pulse energies and scanning speeds, as seen in Fig. 4. It is expected that at speeds higher than $500 \mu \mathrm{m} / \mathrm{s}$ the etch rate starts decreasing [19]. For energies lower than 60 $\mathrm{nJ}$ the etch rate decreased abruptly, meaning the threshold for selective modification is around this energy level.

Focusing the beam deeper into the glass resulted in an increase of the spherical aberrations, which spread out the focused energy and weakened the contrast between irradiated and non-irradiated zones. This effect results in an increase in the etch rate for channels fabricated with parallel polarization.

In addition, the etch rate depends on the separation between scans (Fig. 5). When the laser-affected zones do not overlap, the etch rate is similar to the one obtained for single-scan channels. However, decreasing the separation between scans results in an increase of the stress field around the laser-affected zones, 


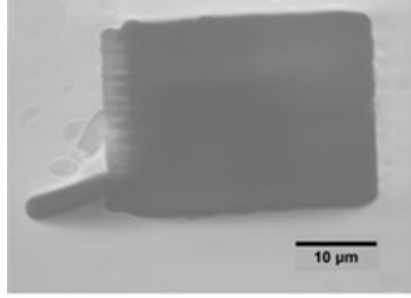

(a)

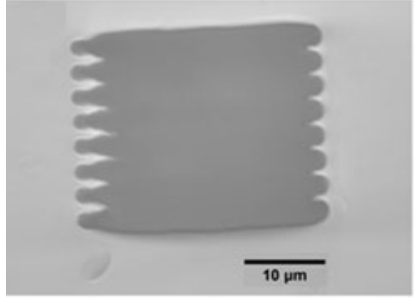

(c)

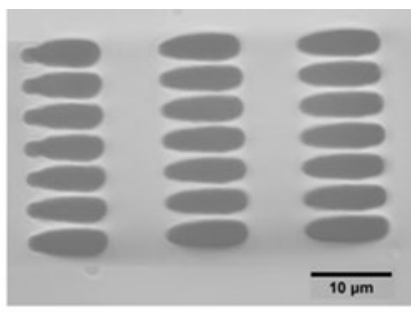

(b)

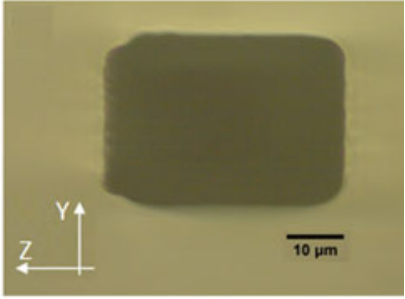

(d)
Fig. 6. Cross-section images of microfluidic channels: (a) channel with microcracks; (b) cappilaric structures; (c) channel with corrugated walls; (d) channel with smooth sidewalls. The indicated reference system is common to all images (the $\mathrm{Z}$ axis direction corresponds to an increasing depth and the $\mathrm{Y}$ axis to the lateral spacing).

which modifies the irradiated volume density and, consequently, increases the etch rate. It is also noticed that the separation along Y plays a more significant role, which is attributed to the elliptical profile of the laser-affected zone that is elongated along the $\mathrm{Z}$ direction.

The channel surface roughness depends mainly on two factors: stress field distribution and scan separation. The formation of laser-affected zones is associated with the generation of stress fields around the irradiated volume [20]. High pulse energies and lower scanning speeds favor stress accumulation, which at extreme conditions (above $300 \mathrm{~nJ}$ ), can induce cracks that propagate over a few microns into the channel surroundings (Fig. 6(a)). On the other hand, if the laser-affected zones are separated by tenths of microns, it is observed that after etching these volumes do not merge (Fig. 6(b)).

By decreasing the scan separation, the volumes start merging, but the sidewalls are still strongly corrugated (Fig. 6(c)). Therefore, it is necessary to continue decreasing the scan separation to obtain smoother sidewalls, while avoiding, at the same time, crack formation (Fig. 6(d)).

The optimum irradiation parameters are thus: beam polarization perpendicular to the scanning direction, scanning speed of $500 \mu \mathrm{m} / \mathrm{s}$, pulse energy of $80 \mathrm{~nJ}$ and scan separation along Y of $1 \mu \mathrm{m}$ and along $\mathrm{Z}$ of $2 \mu \mathrm{m}$. Under these conditions, it was obtained a maximum etching selectivity of 140:1 and a surface roughness of around $100 \mathrm{~nm}$ along $\mathrm{Y}$ and of tenths of nanometers along $\mathrm{Z}$ (Fig. 7). The different surface roughness along $\mathrm{Y}$ and $\mathrm{Z}$ are due to the nanograting alignment. An example of a channel fabricated with these parameters and at initial stages of etching is shown in Fig. 6(d) and in Fig. 7.

For the proposed device, the channel was written $85 \mu \mathrm{m}$ below surface (distance from silica surface to the top of the channel) and with dimensions of $1 \mathrm{~cm}$ in length and $35 \mu \mathrm{m} \times 35 \mu \mathrm{m}$ in cross-section. Because the channel is fabricated directly buried

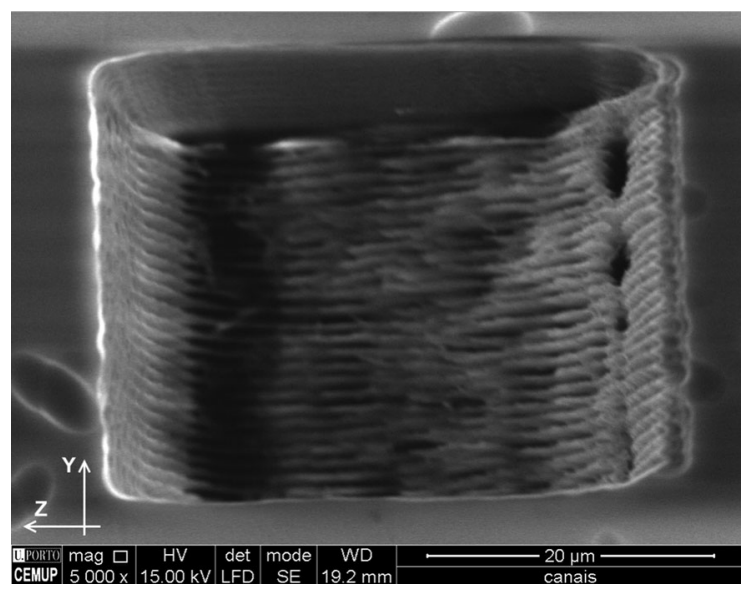

Fig. 7. SEM cross-section image of a microfluidic channel fabricated under optimum exposure conditions, after 30 minutes of etching. The $\mathrm{Z}$ axis direction corresponds to an increasing depth and the $\mathrm{Y}$ axis to the lateral spacing.

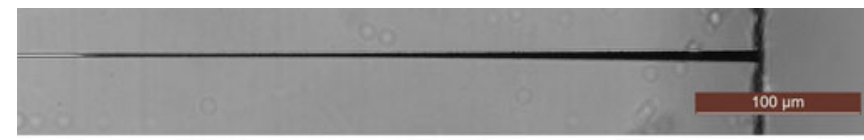

(a)

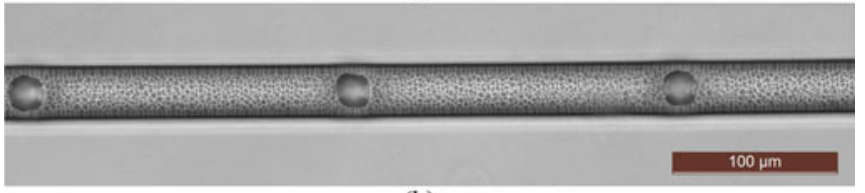

(b)

Fig. 8. Top view image of (a) tapered channel and (b) channel with vertical holes distanced $150 \mu \mathrm{m}$.

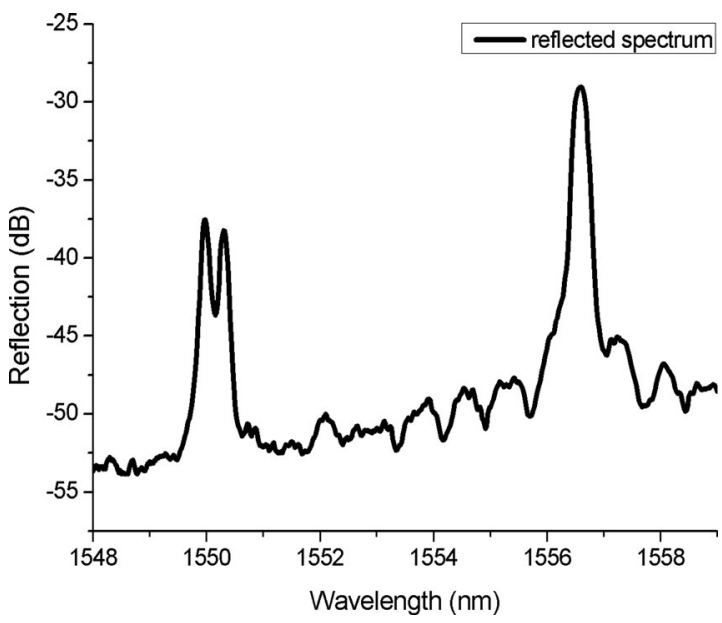

Fig 9. Optical spectrum prior to the etching reaction.

in the fused silica sample, it is necessary to design vertical holes, connecting the channel to the sample surface, for acid diffusion. If the holes are designed at each end of the channel, the obtained channel has the form of a taper (Fig. 8(a)), due to etch rate saturation and lateral etching (the acid starts etching the surrounding material), which results in a non-uniform aspect ratio [19]. Therefore, to avoid this issue, vertical holes were designed periodically with a separation of $150 \mu \mathrm{m}$ (Fig. 8(b)). 

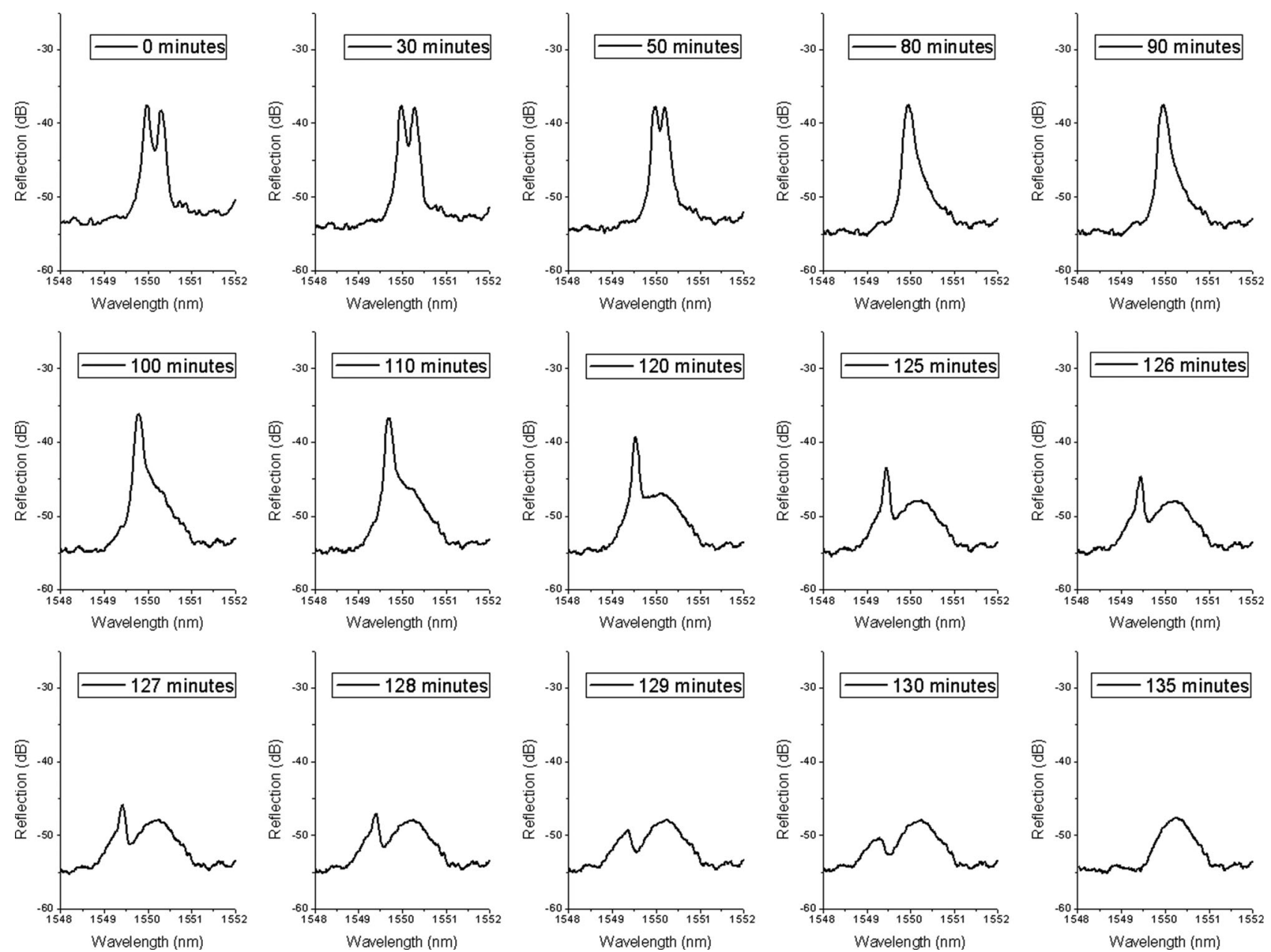

Fig. 10. Optical spectrum of the BGW over etching time.

\section{Fabrication of Bragg Grating Waveguides}

The waveguiding medium was written with beam polarization parallel to the scanning direction, at $250 \mathrm{~nJ}$ pulse energy, $400 \mu \mathrm{m} / \mathrm{s}$ scanning speed and $100 \mu \mathrm{m}$ below the silica surface. These conditions yielded unperturbed optical waveguides with minimum propagation losses, typically of $0.14 \mathrm{~dB} / \mathrm{cm}$, at $1550 \mathrm{~nm}$.

To write the waveguide gratings, the laser beam was modulated with a square-wave function of $40 \%$ duty cycle, as a compromise between grating strength and low propagation loss. The sensing grating was written at a frequency of $746.129 \mathrm{~Hz}$ for resonance at $1550 \mathrm{~nm}$, while the reference grating was written at $742.942 \mathrm{~Hz}$ for a reflection at $1558 \mathrm{~nm}$, which corresponds to modulation periods of $536.1 \mathrm{~nm}$ and $538.4 \mathrm{~nm}$ at $\mathrm{v}=400 \mu \mathrm{m} / \mathrm{s}$ respectively. These conditions resulted in two first-order Bragg gratings. The sensing grating was placed $6.3 \mu \mathrm{m}$ away from the microfluidic channel (distance between the centre of the BGW and the channel sidewall).

\section{E. Monitorisation of Etching Reaction}

To fabricate a highly-sensitive device, the separation between channel and BGW cannot be more than a few microns. This is usually difficult to achieve, because the HF acid may start carving into the $\mathrm{BGW}$, thereby damaging the device. Therefore, the etching reaction has to be monitored in order to control the distance between the two structures.

After polishing, an assembled V-groove coupling block was aligned with the optical waveguide and glued to it. This approach was the only way to maintain the alignment between the optical fiber and the waveguide intact during the etching reaction. To monitor the etching reaction, randomly polarized light from a broadband source (EXFO IQ-2300) was routed through an optical circulator and coupled into the V-groove fiber. The reflected light from the BGWs was passed back to the optical circulator and guided to an optical spectrum analyzer (OSA).

An example of the obtained optical spectrum, before starting the etching reaction, is shown in Fig. 9. It is observed an increase in the birefringence (equal to $3 \times 10^{-4}$ ) of the sensing grating, relatively to the case where there is no channel close to the $\mathrm{BGW}$. This birefringence is related to the presence of the channel modified material that induces stress in the BGW [21].

The optical spectra obtained during the entire etching reaction are depicted in Fig. 10. After 10 minutes of etching, there is no difference in the optical spectrum.

Observations at a microscope revealed that, after this time, only the vertical holes had been etched. After 20 minutes the 


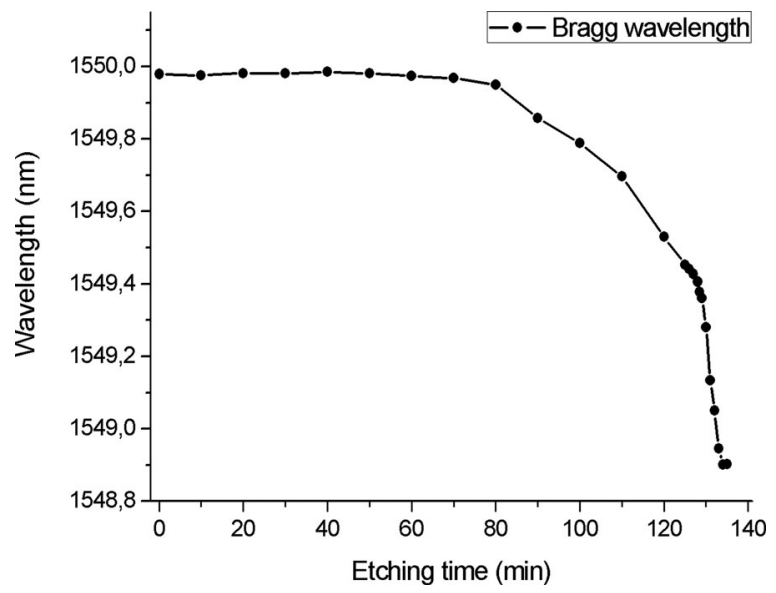

Fig. 11. Bragg wavelength evolution over etching time.

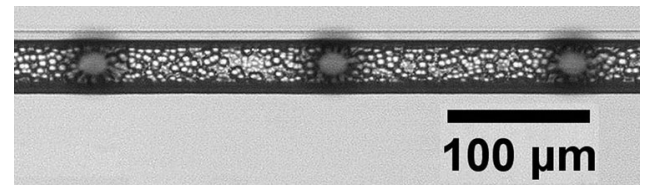

Fig. 12. Top view image of the final device. The BGW and the microfluidic channel are separated by $3.8 \mu \mathrm{m}$ (distance between the centre of the BGW and the channel sidewall).

birefringence starts decreasing, and after 80 minutes it is not measurable with this configuration.

The decrease in birefringence is due to the etching reaction in the microfluidic channel, which relieves the material stress. At this time it was observed that the microfluidic channels had been fully etched [21].

Continuing with the etching reaction, the reflected peak started shifting to wavelengths lower than $1550 \mathrm{~nm}$, as seen in Fig. 11. At this moment, the channel is filled with $\mathrm{HF}$ acid, which has a lower refractive index than fused silica, thereby causing this shift, according to (1). In addition, the HF starts etching the channel surroundings, reducing the gap between channel and BGW; this causes an increase on the overlap between the guided mode and the fluidic medium, leading to a decrease in the effective index and, consequently, on the reflected wavelength. The latter effect is accompanied by a decrease in the grating strength that is more visible after 120 minutes, because the HF acid starts carving into the BGW.

At 135 minutes it is visible a small peak at $1550 \mathrm{~nm}$, which is a result of the grating starting and ending $1 \mathrm{~mm}$ outside the channel. At this time, the etching reaction was completed and the substrate was then cleaned in an ultrasonic bath of deionised water. Subsequent microscope observations revealed that the monitored grating (BGW1) had been erased, while the reference grating (BGW2) was left unperturbed.

A sacrificial waveguide was used to obtain the results of Fig. 10, but a more distant waveguide (Fig. 12) was utilized for characterization of the fluidic refractive index. Initially this BGW was distanced $9.5 \mu \mathrm{m}$ from the channel, but after etching for 135 minutes the gap was reduced to $3.8 \mu \mathrm{m}$ (distances taken from the centre of the BGW to the channel sidewall). For comparison,

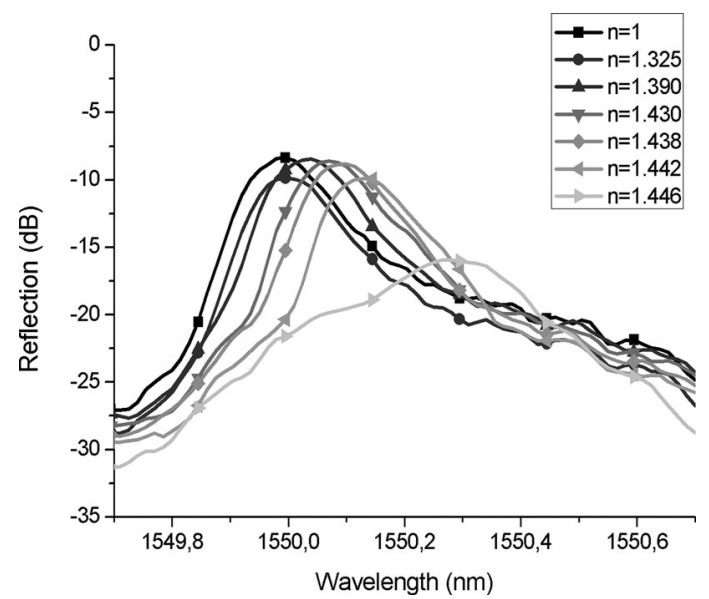

Fig. 13. Reflected spectrum for different fluid refractive indexes.

the guided mode of an unperturbed waveguide has a mode field diameter of $7.1 \mu \mathrm{m} \times 12.3 \mu \mathrm{m}$, along $\mathrm{Y}$ and $\mathrm{Z}$ respectively, which enables interaction between the mode and the channel.

\section{REFRACTIVE InDEX MEASUREMENTS}

Randomly polarized light from a broadband source (EXFO IQ-2300) was routed through an optical circulator and coupled into the waveguide with a single-mode fiber. To decrease Fresnel reflections from the air-silica interface, index matching fluid was used. This experiment used a microfluidic channel and waveguide different from the one used for etching monitoring, that were on the same substrate but not with optical fibers permanently glued to them. The reflected light from the BGW was passed back to the optical circulator and guided to an OSA.

Different index matching fluids from Cargille were used to measure a refractive index of: 1.325, 1.390, 1.430, 1.438, 1.442 and $1.446\left( \pm 0.001\right.$, at $1550 \mathrm{~nm}$ and $\left.25^{\circ} \mathrm{C}\right)$. The fluids were drawn into the channel by capillary forces. After each measurement, the device was rinsed in an ultrasonic bath of acetone. All the measurements were performed with a resolution of $0.05 \mathrm{~nm}$ and without polarization control. The obtained reflected spectrum was normalized relative to the laser spectrum.

An overview image of the reflected spectrum for different refractive indexes is depicted in Fig. 13. Clearly, the resonant behavior of the BGW depends on the fluid refractive index inserted in the channel: as the refractive index increases, the Bragg wavelength shifts towards higher wavelengths. It is also observed that for a refractive index of 1.446 , the propagation loss increased because the index difference between BGW and channel is almost null and the guided mode is less well-confined.

Fig. 14 represents the measured Bragg wavelength as a function of the refractive index of the fluid circulating in the channel. As expected this variation is non-linear. The reference grating is only sensitive to temperature or strain changes and therefore it was confirmed that its Bragg wavelength was independent of the fluid refractive index. While performing the measurements, the room temperature varied by $1^{\circ} \mathrm{C}$, which does not affect significantly the Bragg wavelength. 


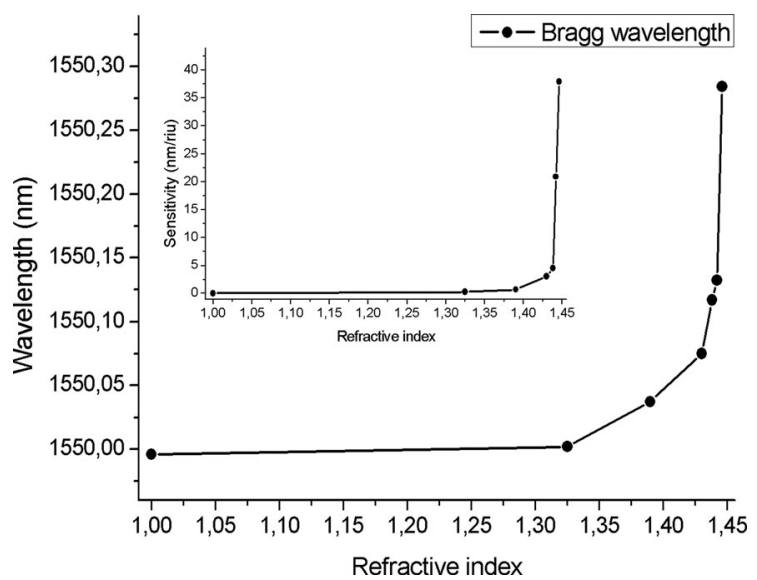

Fig. 14. Bragg wavelength as a function of the fluid refractive index. The inset graph corresponds to the sensitivity.

The refractive index sensitivity, which was obtained by performing the derivative of the curve presented in Fig. 14, is depicted in the inset of the figure. The results show that the sensitivity increases non-linearly as the refractive index increases, having its maximum when the refractive index of the fluid circulating in the channel matches the refractive index of the substrate (fused silica).

The highest sensitivity obtained is of $38 \mathrm{~nm} /$ riu at 1.446 (1550 $\mathrm{nm}$ ), which under these measurement conditions (resolution of $0.05 \mathrm{~nm}$ ) represents a minimum resolvable refractive index variation of approximately $1.3 \times 10^{-3}$.

Similar devices have already been fabricated in optical fibers. For instance, in [22] a refractive index sensitivity of $1394 \mathrm{~nm} / \mathrm{riu}$ at $1.44(1550 \mathrm{~nm})$ was obtained by etching an optical fiber until its core (which contains the BGW) had a diameter of $3 \mu \mathrm{m}$, while in [23] a sensitivity of $300 \mathrm{~nm} /$ riu at $1.455(976 \mathrm{~nm})$ was obtained by side-polishing a fiber so that a Bragg grating could interact evanescently with a liquid analyte. To achieve such sensitivities in planar devices, the distance between channel and BGW needs to be precisely defined which is only possible if the etching reaction is well-controlled: if the etching is stopped when the Bragg peak strength starts decreasing, then the gap between the two elements can be of submicron range.

Further obvious improvements can be implemented by inserting the microfluidic channel close to one arm of a MachZehnder interferometer containing a Bragg grating. The coarse determination of the refractive index change will be retrieved by analyzing the reflection spectrum of the Bragg grating while high resolution measurements are done by analyzing the transmitted interferometric signal (at a different wavelength than that of the BGW resonance). Also, the refractive index sensitivity can be enhanced by fabricating multiple channels surrounding the BGW to increase coupling between the evanescent field and the fluid [3], or by changing the type of the BGW to give a narrower linewidth.

\section{CONCLUSION}

This paper discusses the fabrication of microfluidic channels by femtosecond laser micromachining. An overview of the effect of the irradiation conditions on the channel properties (aspect ratio and surface roughness) is presented: the channels should be fabricated with perpendicular polarization using energies near the threshold for selective etching.

The fabricated channels were integrated with a BGW for monitoring the etching process. A detailed analysis of the etching reaction is provided, enabling careful control of the BGWchannel distance. In sum, (i) as the HF acid fills the channel and the gap channel-BGW decreases, the resonant wavelength increases, and (ii) when the etchant starts carving into the BGW, the reflected peak strength decreases and the reaction has to be stopped. The presented methodology (etching monitorisation) can be used to fine tune the distance between channel and waveguiding medium, which can be of significant use in the fabrication of highly-sensitive optofluidic systems.

Furthermore the proposed scheme also enables the use of the sensing grating as a coarse refractometer, having been obtained a sensitivity of $38 \mathrm{~nm} / \mathrm{riu}$ at $1.446(1550 \mathrm{~nm})$. High resolution refractive index sensors can be accomplished if the set is fabricated in the arms of a Mach-Zehnder interferometer, for example.

\section{REFERENCES}

[1] P. S. Dittrich and A. Manz, "Lab-on-a-chip: Microfluidic in drug discovery," Nature Rev. Drug Discov., vol. 5, no. 3, pp. 210-218, Mar. 2006.

[2] B. Xu, Y. Zhang, H. Xia, W. Dong, H. Ding, and H. Sun, "Fabrication and multifunction integration of microfluidic chips by femtosecond laser direct writing," Lab Chip, vol. 13, no. 9, pp. 1677-1690, May 2013.

[3] D. Hwang, M. Kim, K. Hiromatsu, H. Jeon, and C. Grigoropoulos, "Threedimensional optofluidic devices fabricated by ultrashort laser pulses for high throughput single cell detection and processing," Appl. Phys. A, vol. 96, no. 2, pp. 385-390, Mar. 2009.

[4] G. Whitesides, "The origins and the future of microfluidics," Nature, vol. 442, pp. 368-373, Jul. 2006.

[5] H. Wu, A. Wheeler, and R. N. Zare, "Chemical cytometry on a picoliter scale microfluidic chip," in Proc. Nat. Acad. Sci. USA, vol. 101, pp. 12809-12813, 2004

[6] D. Choudhury, T. Ramsay, R. Kiss, A. Willoughby, L. Paterson, and K. Kar, "A 3D mammalian cell separator biochip," Lab Chip, vol. 12, no. 5, pp. 948-953, Mar. 2012.

[7] K. Sugioka and Y. Cheng, Femtosecond Laser 3D Micromachining for Microfluidic and Optofluidic Applications, 1st ed. Berlin, Germany: Springer, 2014, ch. 3, pp. 19-33.

[8] S. Nolte, M. Will, J. Burghoff, and A. Tuennermann, "Femtosecond waveguide writing: A new avenue to three-dimensional integrated optics," Appl. Phys. A, vol. 77, no. 1, pp. 109-111, Jun. 2003.

[9] Y. Bellouard, A. Said, M. Dugan, and P. Bado, "Fabrication of high-aspect ratio, micro-fluidic channels and tunnels using femtosecond laser pulses and chemical etching," Opt. Express, vol. 12, no. 10, pp. 2120-2129, May 2004.

[10] H. Stone, A. Stroock, and A. Ajdari, "Engineering flows in small devices: Microfluidics towards a lab-on-a-chip," Annu. Rev. Fluid Mech., vol. 36, pp. 381-411, 2004.

[11] B. Lin, Microfluidic-Technologies and Applications, 1st ed. Berlin, Germany: Springer, 2011, pp. 27-68.

[12] D. Choudhury, J. Macdonald, and A. Kar, "Ultrafast laser inscription: perspectives on future integrated applications," Laser Photon. Rev., vol. 8, no. 6, pp. 827-846, Nov. 2014.

[13] V. Maselli, J. R. Grenier, S. Ho, and P. R. Herman, "Femtosecond laser written optofluidic sensor: Bragg grating waveguide evanescent probing of microfluidic channel," Opt. Express, vol. 17, no. 14, pp. 11719-11729, Jul. 2009.

[14] A. Said, M. Dugan, P. Bado, Y. Belloiuard, A. Scoot, and J. Mabesa, "Manufacturing by laser direct-write of three-dimensional devices containing optical and microfluidic networks," Proc. SPIE, vol. 5339, pp. 194-204, 2004. 
[15] R. Osellame, G. Cerullo, and R. Ramponi, Femtosecond Laser Micromachining: Photonic and Microfluidic Devices in Transparent Materials, $1 \mathrm{st}$ ed. Berlin, Germany: Springer, 2012, ch. 14, pp. 389-420.

[16] P. G. Kazansky et al., "“Quill” writing with ultrashort light pulses in transparent materials," Appl. Phys. Lett, vol. 90, no. 15, 2007, Art. no. 151120.

[17] C. Hnatovsky et al., "Fabrication of microchannels in glass using focused femtosecond laser radiation and selective chemical etching," Appl. Phys. A, vol. 84, no. 1/2, pp. 47-61, Apr. 2006.

[18] K. Liu et al., "A high-efficiency three-dimensional helical micromixer in fused silica," Microsys. Technol., vol. 19, no. 7, pp. 1033-1040, Jul. 2013.

[19] S. Ho, P. R. Herman, and J. S. Aitchison, "Single- and multi-scan femtosecond laser writing for selective chemical etching of cross-section patternable glass micro-channels," Appl. Phys. A, vol. 106, no. 1, pp. 5-13, Jan. 2012.

[20] A. Champion, M. Beresna, P. Kazansky, and Y. Bellouard, "Stress distribution around femtosecond laser affected zones: Effect of nanogratings orientation," Opt. Express, vol. 21, no. 21, pp. 24942-24951, Oct. 2013.

[21] L. A. Fernandes, J. R. Grenier, P. R. Herman, J. S. Aitchison, and P. V. S. Marques, "Stress induced birefringence tuning in femtosecond laser fabricated waveguides in fused silica," Opt. Express, vol. 20, no. 22, pp. 24103-24114, Oct. 2012.

[22] A. N. Chryssis, S. M. Lee, S. B. Lee, S. S. Saini, and M. Dagenais, "High sensitivity evanescent field fiber Bragg grating sensor," IEEE Photon. Technol. Lett., vol. 17, no. 6, pp. 1253-1255, Jun. 2005.

[23] K. Schroeder, W. Ecke, R. Mueller, R. Willsch, and A. Andreev, "A fibre Bragg grating refractometer," Meas. Sci. Technol., vol. 12, no. 7 , pp. 757-764, Jul. 2001

João M. Maia was born in Porto, Portugal in 1993.He received the B.Sc. degree in technological Physics and the M.Sc. degree in physics engineering, both from the University of Porto, Portugal, in 2014 and 2016, respectively. Since September 2016, he is working toward the Ph.D. degree in physics with the University of Porto .In 2015, he joined the Centre for Applied Photonics of INESC TEC (Porto, Portugal), where he is working as a Researcher. His current research interests include fabrication of optofluidic devices by femtosecond laser micromachining for chemical and biosensing.
Vítor A. Amorim was born in Barcelos, Portugal in 1993. He received the B.Sc. degree in technological physics andthe M.Sc. degree in physical engineering from the University of Porto, Portugal in 2014 and 2016, respectively on the fabrication of integrated optical devices in fused silica using the femtosecond laser direct writing technique. Since 2016, he is working toward the Ph.D. degree in physics in the aforesaid institution. He joined the Centre for Applied Photonics of INESC TEC in 2015, where he is currently enrolled in research activity. His current research interests include integrated optical devices and monolithic optofluidic devices fabricated by femtosecond laser direct writing techniques for bio and chemical sensing.

Daniel Alexandre was born in Viseu, Portugal in 1976. In 1999, he received the degree in electrotechnics engineering from the University of Trás-os-Montes e Alto Douro, Portugal, Portugal. He received the M.Sc. degree in optoelectronics and lasers and the Ph.D. degree in physics from the University of Porto, Portugal, in 2003 and 2011, respectively. His Ph.D. research programme was conducted under a strong collaboration with the Centre for Applied Photonics (CAP) at INESC TEC (Porto, Portugal) and it involved the development and characterization of passive and active integrated optical devices using hybrid sol-gel and laser direct writing. He started teaching as a Monitor in the Physics Department of the University of Trás-os-Montes e Alto Douro in March 1999, where he was appointed as an Assistant Professor, since 2011. Since 2002, he develops research activity in the CAP at INESC TEC. His current research interests include integrated optical devices and microfluidics, Bragg gratings, integrated optical sensors, and laser direct writing techniques for integrated optics and microfabrication.

Paulo V. S. Marques is an Assistant Professor in the Physics and Astronomy Department, Faculty of Science of Porto University, Portugal. Since October 2009. he is the Coordinator of the Center of Applied Photonics of INESCTEC and the Director of the Micro and Nanofabrication Center of Porto University (CEMUP MNTEC, May 2013 ). His current research interests include optical sensors, femtosecond laser direct writing techniques for integrated optics and microfabrication in general, microfluidics, Bragg gratings, and active devices. He has published 4 world patents (patent family of 32), more than 100 scientific papers in international magazines and conferences and three book chapters. 